\title{
INFLUENCE OF POLISHING SYSTEM ON COLOR CHANGES FOR A NANOIONOMER RESTORATIVE MATERIAL AFTER IMMERSION IN A COFFEE-BASED SOLUTION
}

\author{
ELLYZA HERDA*, PUTRA AKA, SITI TRIAMININGSIH
}

Department of Dental Materials, Faculty of Dentistry, Universitas Indonesia, Jakarta, Indonesia. Email: ellyza_herda@yahoo.com

Received: 15 August 2018, Revised and Accepted: 20 March 2019

\begin{abstract}
Objective: In this study, we aim to assess a polishing system's influence on the color change of a nanoionomer after immersion in a coffee-based solution.

Methods: The specimens were divided into four groups, of which three of them were polished using Sof-Lex XT, PoGo, or OptraPol. However, the samples in the fourth group were unpolished. The color was then determined using a spectrophotometer and color changes were evaluated using a CIELAB system. The data were analyzed using one-way ANOVA and paired samples t-test systems.

Results: While the lightness $\left(\mathrm{L}^{*}\right)$ value significantly decreased $(\mathrm{p}<0.05)$ after the polishing procedure, the red-green coordinate $\left(\mathrm{a}^{*}\right)$ and yellow-blue coordinate $\left(b^{*}\right)$ values did not show any significant change for any of the groups. The samples that were polished using OptraPol demonstrated the smallest color change after polishing; moreover, for this group, the color changes were significantly different from those that were observed for PoGo and Sof-Lex XT groups. After immersing into a coffee-based solution, there were significant changes observed for the $\mathrm{L}^{*}$, $\mathrm{a}^{*}$, and $\mathrm{b}^{*}$ values ( $<<0.05$ ) for all the groups that were studied. Importantly, the color changes that were observed for the OptraPol group were not statistically significant compared to those that were observed for PoGo and the unpolished groups.
\end{abstract}

Conclusion: The immersion of unpolished and polished nanoionomers in a coffee-based solution will result into color changes and the smallest variations, in this case, occurred for the nanoionomer that was polished using OptraPol.

Keywords: Nanoionomer, Polishing system, Coffee, Discoloration.

(C) 2019 The Authors. Published by Innovare Academic Sciences Pvt Ltd. This is an open access article under the CC BY license (http://creativecommons. org/licenses/by/4. 0/) DOI: http://dx.doi.org/10.22159/ijap.2019.v11s1.XX

\section{INTRODUCTION}

Nanoionomers were restorative materials comprising a resinmodified glass ionomer cement (RM-GIC) that contains nanosized filler particles. Such types of materials combine the advantages of a GIC along with those of a nanofiller technology. This leads to an esthetically superior polishing capability; moreover, the size of the filler particles may influence the strength, optical characteristics, as well as abrasion tenacity for the process. Thus, for developing nanoionomers, nanomers and nanoclusters that contain fluoroaluminosilicate glass as the filler are used [1]. This helps develop esthetic structures along with the fluoro-releasing advantages of the GIC [2].

A very challenging problem posed by esthetic materials is finishing and polishing of restoration surfaces. If there is improper polishing, a coarse surface may lead to both retention of food debris and a dirty and stained restoration surface [3]. Therefore, polishing is used to make restoration look more natural and harmonious along with the adjacent tooth structures, particularly for regularity and shine of the surface. Moreover, a smooth surface may decrease plaque retention, thus facilitating good oral hygiene [4]. Therefore, an appropriate polishing technique is required for producing smoother surfaces and for minimizing staining and color changes during restoration.

Bouvier et al. studied the RM-GIC and demonstrated that, compared to treatments with diamond-impregnated and tungsten carbide polishing systems, polishing with a flexible polishing disc that is coated with aluminum oxide results in a smoother surface [5]. When Mallya et al. were studying nanoionomers, they observed that restorative procedures required only a Mylar matrix without finishing and polishing, which helped produce the smoothest surface. If finishing and polishing were performed later, the smoothness decreased in the following order: Flexible disc coated using aluminum oxide $>$ tungsten carbide $>$ diamond-finishing point [6].

Coffee is one of the most frequently consumed drinks and, after Brazil and Vietnam, Indonesia is the third largest producer of coffee in the world. On an average, coffee consumption in Indonesia has reached $1.2 \mathrm{~kg}$ per capita per year [7]. In fact, the habit of drinking coffee can lead to discoloration for restorations. Ertaş et al. stated that discoloration caused by coffee occurs because the materials absorb coloring agents [8]. Chan et al. stated that discoloration due to coffee was significantly higher than that caused by tea [9]. A similar result was reported by Gupta et al. who stated that the intensity of coffee stains was higher than that of tea stains [10].

Based on manufacturers' instructions, nanoionomers were best polished using conventional polishing instruments [11]. To the best of our knowledge, there have been no previous studies on the effect of different polishing systems on the color changes of nanoionomer materials due to immersion in coffee-based solutions.

\section{METHODS}

Using a cylindrical stainless-steel mold $(6 \mathrm{~mm}$ in diameter and $2 \mathrm{~mm}$ in height), 24 specimens were molded. Then, using a Mylar strip, a nanoionomer (Ketac N 100, 3M ESPE, USA) was filled and flattened on the surface. Later, a microscopic slide along with a $1 \mathrm{~kg}$ load was placed on that the ionomer. Then, the material was cured using a light-curing unit (HILUX LEDMAX 450, Turkey) for 20 s. Subsequently, the specimens were collected from the mold, 
immersed in aqua bidest, and stored in an incubator at a controlled temperature of $37^{\circ} \mathrm{C}$ for $24 \mathrm{~h}$.

Using a digital spectrophotometer (VITA Easyshade ${ }^{\circledR}$, Germany), the initial color determination was performed with the probe's being placed perpendicular to the specimen's surface while gently touching it to obtain the $\mathrm{L}^{*}{ }_{1}, \mathrm{a}^{*}{ }_{1}$, and $\mathrm{b}^{*}{ }_{1}$ data. Then, the specimens were divided into four groups, of which three were polished using Sof-Lex XT Superfine, PoGo Point, or OptraPol Large Flame; however, the fourth group remained unpolished. Note that each group contained six specimens.

Furthermore, the polishing material was then placed on a contraangle handpiece (NSK, Japan) and a polishing simulator. It was then connected to a micromotor (Saeshin Strong 90, Korea). Polishing was then performed for $20 \mathrm{~s}$ at 35,000 rpm with light hand pressure. When polishing, using a rotation movement, polishing with Sof-Lex XT Superfine was performed without water; using a planar movement, polishing with PoGo Point was performed without water; and, using a planar movement, polishing with OptraPol Large Flame was performed with water. Subsequently, after polishing, the specimens were rinsed using aqua bidest in an ultrasonic cleaner for $4 \mathrm{~min}$ and then dried.

The color was then determined again for obtaining the $\mathrm{L}_{2}^{*}, \mathrm{a}^{*}{ }_{2}$, and $\mathrm{b}^{*}{ }_{2}$ data using the same methods that were applied for obtaining the first set of data. Later, from each group, one specimen was randomly selected for surface morphology observations along with a scanning electron microscope (SEM) (JEOL, JSM-6510LA, Japan) at $\times 1500$ magnification at $20 \mathrm{kV}$.

In different coffee-based solutions (i.e., in a beaker glass containing $150 \mathrm{ml}$ solution), the specimen groups were immersed until the complete specimen surface was submerged without it coming in contact with the bottom of the glass. The coffee-based solution was prepared using Robusta coffee powder (Nescafe Classic), which was mixed with boiled water in a beaker and the powder-to-coffee ratio was maintained at $2 \mathrm{~g}$ for $150 \mathrm{ml}$. Using a filter paper, this solution was mixed well and the temperature of the coffee was maintained at $37^{\circ} \mathrm{C}$ when the specimens were placed inside. For 7 days, staining was performed with coffee-based solutions that were regularly changed. Before placing samples in newer coffeebased solutions, the samples were rinsed with $100 \mathrm{ml}$ aqua bidest for $1 \mathrm{~min}$. According to Guler et al., an average person drinks 3.2 cups of coffee per day; moreover, for each cup, they spend over 15 min [12] Therefore, immersing for 7 days simulates the coffee consumption for a 7 -month period [13].

To obtain the $\mathrm{L}^{*}, \mathrm{a}^{*}{ }_{3}$, and $\mathrm{b}^{*}{ }_{3}$ data, after immersion in coffee-based solutions (staining), the specimens' color was evaluated once more. Then, to obtain the $\Delta \mathrm{E}^{*}, \Delta \mathrm{L}^{*}, \Delta \mathrm{a}^{*}$, and $\Delta \mathrm{b}^{*}$ values, all the $\mathrm{L}^{*}, \mathrm{a}^{*}$, and $\mathrm{b}^{*}$ data were processed for the normal and homogenous data, which were tested for each polishing group using the one-way ANOVA test, followed by post hoc Tukey's honestly significant difference test. However, for the non-normal and non-homogenous data, the Kruskal-Wallis test, followed by the post hoc Mann-Whitney U test, was used. The test was aimed to determine differences in the color values that were obtained between the polishing groups due to polishing intervention and the specimen groups that were immersed in a coffee-based solution. To measure the significance of different values of lightness, red-green coordinates and yellow-blue coordinates that were obtained for the polishing groups before and after polishing as well as for all specimen groups before and after immersion in a coffee-based solution, a paired sample t-test was performed.

\section{RESULTS AND DISCUSSION}

To obtain the color change value $\left(\Delta \mathrm{E}^{*}\right), \Delta \mathrm{E}^{*}{ }_{1}$ and $\Delta \mathrm{E}^{*}{ }_{2}$ were analyzed with $\Delta \mathrm{E}^{*}$ presenting a color change for nanoionomers before and after being polished. For the polished groups, the $\Delta \mathrm{E}^{*}{ }_{2}$ values indicated color changes in nanoionomers before and after immersion and polishing procedures. The unpolished group demonstrated a color change before and after immersion. However, for the other elements, namely $\Delta \mathrm{L}^{*}, \Delta \mathrm{a}^{*}$, and $\Delta \mathrm{b}^{*}$, the analyzed values were the same. Table 1 lists the color change values obtained for the nanoionomers before and after polishing $\left(\Delta \mathrm{E}^{*}{ }_{1}\right)$.

In nanoionomers, polishing intervention caused color changes using the polishing procedure with PoGo, which caused the largest variations. However, polishing with OptraPol led to the smallest color changes, and statistical analysis indicated that color changes that occurred after polishing with Sof-Lex XT Superfine were not very different from those observed using PoGo ( $\mathrm{p}=0.522)$. However, there were significant differences between the Sof-Lex XT Superfine and OptraPol groups $(\mathrm{p}<0.05)$, and color change values obtained using PoGoand OptraPol showed significant differences.

In nanoionomers, color changes occurred in both the polished and unpolished groups after immersion in a coffee-based solution. Table 2 lists the corresponding color change values $\left(\Delta \mathrm{E}_{2}^{*}\right)$.

The nanoionomers that were polished using OptraPol demonstrated the smallest color changes, whereas those that were treated with SofLex XT Superfine demonstrated the largest ones. During statistical analysis, the color changes in the unpolished group were found to be not as significantly different as those found for the polished groups. Moreover, the Sof-Lex XT Superfine group was significantly different from the PoGo and OptraPol groups $(\mathrm{p}<0.05)$, which did not show any statistically important differences between each other.

Furthermore, the changes in lightness value were analyzed. While a higher lightness value indicated a lighter result, a lower value indicated a darker result. Table 3 lists changes in lightness values for nanoionomers before and after the polishing procedure.

After the polishing procedure, the nanoionomers became darker with the largest change occurring for materials that were polished using Sof-Lex XT Superfine, whereas the smallest change occurred for

Table 1: Average color changes in nanoionomers after the polishing procedure

\begin{tabular}{ll}
\hline Specimen group & $\Delta \mathbf{E}^{*}{ }_{\mathbf{1}}$ (Average $\pm \mathbf{S D}$ ) \\
\hline Mylar strip & - \\
Sof-Lex XT superfine & $3.97 \pm 2.05$ \\
PoGo & $4.01 \pm 1.32$ \\
OptraPol & $1.89 \pm 1.04$ \\
\hline
\end{tabular}

SD: Standard deviation

Table 2: Average color change values for nanoionomers after immersion in a coffee-based solution

\begin{tabular}{ll}
\hline Specimen group & $\Delta \mathbf{E}_{2}^{*}$ (Average $\left.\pm \mathbf{S D}\right)$ \\
\hline Mylar strip & $24.26 \pm 1.14$ \\
Sof-Lex XT superfine & $29.27 \pm 4.02$ \\
PoGo & $23.70 \pm 3.15$ \\
OptraPol & $20.00 \pm 2.89$ \\
\hline
\end{tabular}

SD: Standard deviation

Table 3: Average $L^{*}$ changes for nanoionomers after the polishing procedure (average \pm SD)

\begin{tabular}{llll}
\hline Specimen group & $\mathbf{L}^{*}{ }_{\mathbf{1}}$ & $\mathbf{L}^{*}{ }_{\mathbf{2}}$ & $\Delta \mathbf{L}^{*}{ }_{\mathbf{1}}$ \\
\hline Sof-Lex XT superfine & $70.52 \pm 2.15$ & $46.73 \pm 1.00$ & $-23.78 \pm 2.61$ \\
PoGo & $70.80 \pm 0.75$ & $51.12 \pm 0.59$ & $-19.68 \pm 2.57$ \\
OptraPol & $72.70 \pm 0.51$ & $55.63 \pm 0.84$ & $-17.07 \pm 225$ \\
\hline
\end{tabular}

L*: Lightness, SD: Standard deviation 
OptraPol-treated materials. Based on statistical analysis, no significant differences were found between the three studied groups when the lightness decreased after polishing. However, for all the three groups, the lightness values considerably differed before and after polishing.

Furthermore, a decrease in lightness value occurred after immersion, which was indicated by the resultant darker color. Table 4 lists differences in lightness values for nanoionomers before and after immersion in a coffee-based solution $\left(\Delta \mathrm{L}^{*}{ }_{2}\right)$.

Interestingly, the largest decrease in lightness value occurred for the control group, followed by the Sof-Lex XT Superfine, PoGo, and OptraPol groups. Such decreases did not significantly differ from those that were observed for the Sof-Lex XT Superfine and PoGo groups; however, they were considerably different from those that were observed the OptraPol group $(\mathrm{p}<0.05)$. Moreover, decreases in lightness value for nanoionomers that were polished using Sof-Lex XT Superfine differed significantly from decreases observed for PoGo and OptraPol groups, which did not differ much from each other. Overall, for all the studied groups, lightness values before and after immersion in a coffee-based solution were significantly different.Table 5 lists the changes in $\mathrm{a}^{*}$ after the polishing procedure.

For polished nanoionomers, the values of red-green coordinates remained in the positive range, although there was some variation. For nanoionomers polished using Sof-Lex XT Superfine, colors moved toward the red direction, whereas the opposite behavior was observed for materials polished using PoGoand OptraPol. However, generally, the red-green coordinates of the nanoionomers that were studied did not considerably change after polishing. Furthermore, for any of the samples, polishing using three different materials did not lead to significant changes in the red-green coordinates.

However, after immersion in a coffee-based solution, the $a^{*}$ value increased for all the groups that were studied, which indicated a redder color. Table 6 lists changes in red-green coordinate values for nanoionomers after immersion in a coffee-based solution $\left(\Delta \mathrm{a}_{2}{ }_{2}\right)$.

Table 4: Average differences in $\mathrm{L}^{*}$ values of nanoionomers after immersion in a coffee-based solution (average $\pm S D$ )

\begin{tabular}{llll}
\hline Specimen group & $\mathbf{L}^{*}{ }_{2}$ & $\mathbf{L}^{*}{ }_{3}$ & $\Delta \mathbf{L}^{*}$ \\
\hline Mylar strip & $74.10 \pm 0.34$ & $52.70 \pm 1.00$ & $-21.40 \pm 2.46$ \\
Sof-Lex XT superfine & $70.52 \pm 2.15$ & $46.73 \pm 1.00$ & $-23.78 \pm 2.61$ \\
PoGo & $70.80 \pm 0.75$ & $51.12 \pm 0.59$ & $-19.68 \pm 2.57$ \\
OptraPol & $72.70 \pm 0.51$ & $55.63 \pm 0.84$ & $-17.07 \pm 2.25$ \\
\hline
\end{tabular}

L*: Lightness, SD: Standard deviation

Table 5: Average differences in $a^{*}$ for nanoionomers after the polishing procedure (average $\pm \mathrm{SD}$ )

\begin{tabular}{llll}
\hline Specimen group & $\mathbf{a}^{*}$ & $\mathbf{a}^{*}{ }$ & $\Delta \mathbf{a}^{*}$ \\
\hline Sof-Lex XT superfine & $4.88 \pm 0.26$ & $5.25 \pm 0.28$ & $0.37 \pm 0.69$ \\
PoGo & $4.88 \pm 0.26$ & $4.70 \pm 0.54$ & $-0.18 \pm 1.32$ \\
OptraPol & $4.88 \pm 0.26$ & $4.50 \pm 0.15$ & $-0.38 \pm 0.36$ \\
\hline
\end{tabular}

SD: Standard deviation, a*: Red-green coordinate

Table 6: Average differences in a* values for nanoionomers after immersion in a coffee-based solution (average \pm SD)

\begin{tabular}{llll}
\hline Specimen group & $\mathbf{a}^{*}{ }_{2}$ & $\mathbf{a}^{*}$ & $\Delta \mathbf{a}^{*}$ \\
\hline Mylar strip & $4.88 \pm 2.26$ & $14.70 \pm 0.31$ & $9.82 \pm 0.76$ \\
Sof-Lex XT superfine & $5.25 \pm 0.28$ & $17.73 \pm 0.81$ & $12.48 \pm 1.93$ \\
PoGo & $4.70 \pm 0.54$ & $15.10 \pm 0.65$ & $10.40 \pm 2.60$ \\
OptraPol & $4.50 \pm 0.15$ & $13.17 \pm 0.54$ & $8.67 \pm 1.51$ \\
\hline
\end{tabular}

SD: Standard deviation, a*: Red-green coordinate
After immersion in the control group, the red-green value that was measured was significantly different from that for the group that was polished using Sof-Lex XT Superfine; however, it did not vary much compared to the results that were obtained for the PoGo and OptraPol groups. Nevertheless, the group that was polished using Sof-Lex XT Superfine showed a significantly different color change compared to the OptraPol group. However, this change was not observed when compared to the PoGo group. Thus, the change in the $a^{*}$ value after immersion between the OptraPol and control groups $(\mathrm{p}=0.796)$ was not very different. Furthermore, for all the studied groups, the red-green coordinate values of nanoionomers after immersion in a coffee-based solution considerably differed from the values that were measured before immersion.

For the different specimens, the changes in yellow-blue coordinates were also analyzed. Table 7 lists the values obtained before and after polishing.

After polishing, the changes in yellow-blue coordinates ( $\left.b^{*}\right)$ exhibited a similar pattern of decrees in value for all the three groups. However, the coordinates remained in the positive range, resulting in a less yellowish color change. For all the samples, after polishing, the changes in yellowblue value were similar, and the yellow-blue value was not significantly affected through the polishing procedure.

Moreover, in the nanoionomers, a color occurred after immersion in a coffee-based solution and for all groups, the color became less yellowish. Table 8 lists the changes in yellow-blue value for the nanoionomers after immersion in a coffee-based solution $\left(\Delta \mathrm{b}_{2}^{*}\right)$.

Importantly, the most significant changes occurred in the group that was polished using Sof-Lex XT Superfine, followed by PoGo, OptraPol, and unpolished groups. For the unpolished group, the decrease in yellow color was significantly different compared to that for the groups that were polished using Sof-Lex XT Superfine and PoGo group; however, it was not significantly different compared to that for the OptraPol group. In the group that was polished using Sof-Lex XT Superfine, the decrease in yellow color was significantly different compared to that for the OptraPol group. However, it was not significantly different compared to that for PoGo group. Interestingly, the results for the PoGo and OptraPol groups were considerably different. Furthermore, the yellow-blue coordinate values of the nanoionomers significantly differed before and after immersion in the coffee-based solution.

In this study, to determine surface morphology, SEM images were considered for nanoionomer samples from each group, as shown in Fig. 1. Moreover, digital images of all the nanoionomer specimens were taken to see the level of cracking on all surfaces (Fig. 2).

Table 7: Average differences in $b^{*}$ values for nanoionomers after the polishing procedure (average \pm SD)

\begin{tabular}{llll}
\hline Specimen group & $\mathbf{b}^{*}{ }_{1}$ & $\mathbf{b}^{*}{ }_{2}$ & $\Delta \mathbf{b}^{*}{ }_{1}$ \\
\hline Sof-Lex XT superfine & $47.34 \pm 0.30$ & $47.03 \pm 0.63$ & $-0.31 \pm 1.54$ \\
PoGo & $47.34 \pm 0.30$ & $46.57 \pm 0.59$ & $-0.78 \pm 1.44$ \\
OptraPol & $47.34 \pm 0.30$ & $46.68 \pm 0.34$ & $-0.66 \pm 0.84$ \\
\hline
\end{tabular}

b*: Yellow-blue coordinate, SD: Standard deviation

Table 8: Average $b^{*}$ value changes for nanoionomers after immersion in a coffee-based solution

\begin{tabular}{llll}
\hline Specimen group & $\mathbf{b}^{*}{ }_{2}$ & $\mathbf{b}^{*}{ }_{3}$ & $\Delta \mathbf{b}^{*}{ }_{2}$ \\
\hline Mylar strip & $47.34 \pm 0.30$ & $41.65 \pm 0.72$ & $-5.69 \pm 1.77$ \\
Sof-Lex XT superfine & $47.03 \pm 0.63$ & $35.65 \pm 1.21$ & $-11.38 \pm 3.48$ \\
PoGo & $46.57 \pm 0.59$ & $38.65 \pm 0.38$ & $-7.92 \pm 0.67$ \\
OptraPol & $46.68 \pm 0.34$ & $40.92 \pm 0.54$ & $-5.77 \pm 1.27$ \\
\hline
\end{tabular}

b*: Yellow-blue coordinate, SD: Standard deviation 


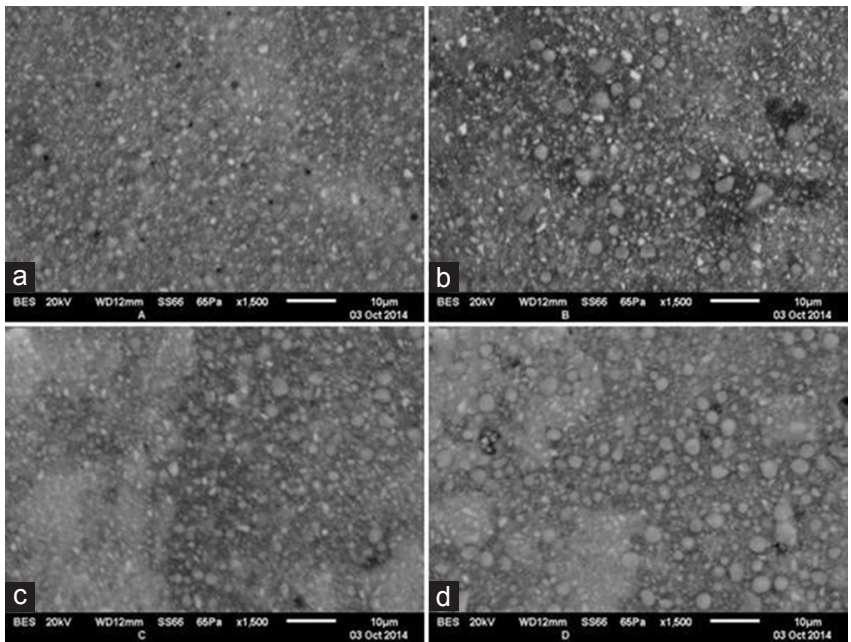

Fig. 1: Micrograph images of nanoionomers at $\times 1500$ magnification. (a) Unpolished nanoionomer; (b) nanoionomer polished using Sof-Lex XT Superfine; (c) nanoionomer polished using PoGo; and (d) nanoionomer polished using OptraPol

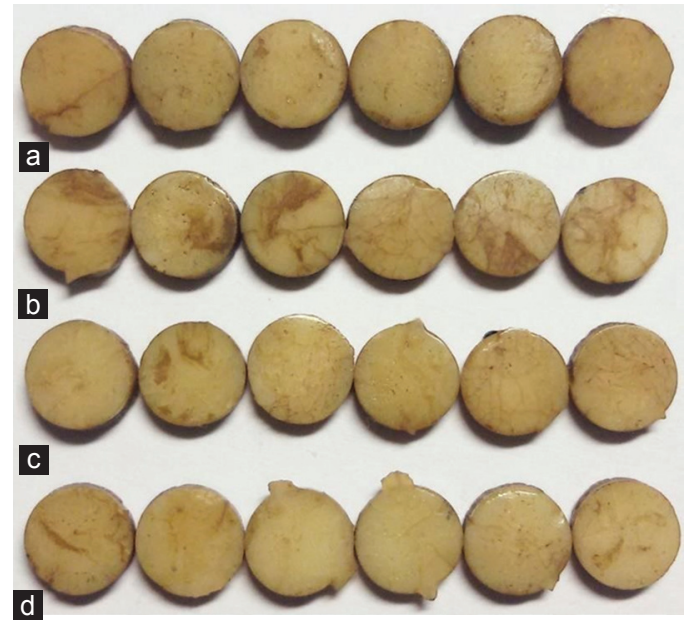

Fig. 2. Nanoionomer specimens after immersion in a coffee-based solution: (a) Unpolished nanoionomer; (b) nanoionomer polished using Sof-Lex XT Superfine; (c) nanoionomer polished using

PoGo; and (d) nanoionomer polished using OptraPol

In Fig. 1a, the exposed fillers on the unpolished nanoionomer surface seemed to be very small and did not protrude to the surface; however, they were evenly dispersed in the matrix. Among all the nanoionomer groups, the surface seemed to be the smoothest. In Fig. 1b, the nanoionomer polished using Sof-Lex XT Superfine exhibited the most inhomogeneous and coarse surface among all the nanoionomer groups that were studied. There were certain areas on the surface of this specimen that contained very exposed fillers. Furthermore, certain fillers were even detached, leaving hollow parts and exposing the matrix. However, other areas contained unexposed fillers; therefore, a portion of the matrix was present on the surface. In Fig. 1c, the nanoionomer that was polished using PoGo had a non-homogenous surface. There were certain areas on the surface of the specimen that seemed to have large exposed fillers, whereas there were still unexposed fillers in other areas, leaving a considerable amount of matrix on the surface. In this group, the fillers seemed to be irregular, with various sizes, but no detached fillers were observed. Overall, the surface seemed to be smoother compared to that for the control group but coarser compared to those of the other polished groups. In Fig. 1d, the nanoionomer polished using OptraPol seemed to have fillers that exhibited regular shapes and various sizes with the largest fillers spread evenly across the surface. For such groups, the exposed fillers on the surface seemed to be the largest among all the studied groups; however, the fillers were still attached to the matrix. For such cases, the surface seemed to be coarser compared to that of the nanoionomer that was polished using PoGo and the unpolished material due to the amount of exposed fillers on the surface.

The digital images of the specimens demonstrated that, for all the nanoionomers, there were differences in the level of cracks on the surface for the studied groups (Fig. 2). The number of cracks on the surface varies in the following order: Sof-Lex XT Superfine $>$ PoGo $>0$ ptraPol $>$ Mylar strip. Furthermore, additional color changes did occur in the cracked areas compared to their surroundings.

\section{DISCUSSION}

Our results demonstrated that color changes did occur in nanoionomers after polishing. Moreover, these changes were also observed after immersion in a coffee-based solution for 7 days. For all the intervention and control groups, these changes were observed. However, after polishing, only the nanoionomer was treated using OptraPol, which exhibited the most clinically acceptable color change.

After immersion in a coffee-based solution, the color changes that occurred were not clinically acceptable for any of the groups. However, the color changes varied in the following order: OptraPol $<\mathrm{PoGo}<\mathrm{Mylar}$ strip $<$ Sof-Lex XT Superfine.

There were significant differences in lightness that was observed for all the groups before and after the polishing procedure may be due to the changes in the surface morphology, which became coarser after polishing. Note that, compared to a smooth surface, a coarse surface will have a lower lightness value when measured using a spectrophotometer [14]. In 2012, Bala et al. reported such increases in the coarseness of nanoionomer surfaces after polishing [15]. Furthermore, the polishing motion was found to contribute to the final result [6]. In this study, the Sof-Lex XT Superfine material, which contained the second smallest particles (after PoGo) and the most lenient abrasive substance, produced the coarsest surface morphology. This was possibly due to the polishing motion (rotation) used for the Sof-LexXT Superfine group. In addition to the polishing motion, water might play a role for producing smoother surfaces. The lowest number of cracks on the surface was exhibited by the group that was polished using OptraPol and water. Such polishing material also contained the same ingredient as PoGo, namelydiamond; it had a larger particle size too. Water served as a cooling agent that prevented both dehydration of polished nanoionomers and formation of cracks on restoration surfaces [16].

For all the studied groups, the differences in red-green and yellow-blue coordinates before and after polishing procedures were not statistically significant, which demonstrate that polishing only affected the lightness value but not the red-green and yellow-blue coordinates.

A decrease in lightness value after immersion in a coffee-based solution happened due to absorption and adsorption of coloring agents by coffee that was dissolved in water [17]. The hydroxyethylmethacrylate (HEMA) matrix present in the nanoionomer exhibited hydrophilic properties, which caused rapid water absorption that could ease the staining process. Furthermore, the coarse restoration surface, which contained an uneven placement of filler particles on it, facilitated the adsorption of color agents [18]. The coloring agents in coffee that caused a decrease in lightness value were tannin and melanoidin, which also created a brown stain $[19,20]$.

For all groups, after immersion in a coffee-based solution, an increase in reddish color (and decrease in yellowish color or a tendency toward blue) occurred, which might have happened due to the absorption and adsorption of anthocyanin present in the coffee. Anthocyanin, a 
water-soluble chemical substance that specifically has a red-blue color, belongs to the flavonoid group; it is a polyphenol molecule [21].

In this study, to observe if there is a correlation between the surface quality and color change that occurs after a material's immersion in a coffee-based solution, micrographs and digital photographs of nanoionomer surfaces were analyzed.

Our results demonstrated that unpolished groups exhibited the smoothest surface with the lowest number of cracks. However, these groups experienced the largest color change after the immersion procedure because these groups had the highest amount of HEMA matrix on the surface. This also enabled an easier absorption of the coloring agent.

The nanoionomers that were polished using Sof-Lex XT Superfine exhibited a non-homogenous surface with detached fillers. They also created hollow parts on it and other areas that contained HEMA matrix; thus, among all the studied groups, this group visually had the highest number of cracks on the specimens' surface. This may be due to the rotation movement that was used during the polishing procedure, particularly for this group, which could have led to deep abrasion in specific areas. The hollow parts that were developed due to the detached fillers created a coarser surface that eased the stain's adsorption; however, the HEMA matrix left on the surface allowed absorption of the coloring agent. Water's absence caused cracks on the surface due to heat-induced dehydration during the polishing procedure. The combination of a coarse surface, presence of HEMA matrix on certain areas of the surface, and cracks contributed to the significant color changes that occurred in this group.

Furthermore, the nanoionomer polished using PoGo exhibited a nonhomogenous surface with certain fillers that were exposed (but not detached) in certain areas. Furthermore, a significant amount of HEMA matrix was present in others. The surface polished using PoGo was the smoothest of all. However, it did not positively contribute to the color tenacity of this group after immersion in a coffee-based solution because the smooth surface was caused by the presence of HEMA matrix that covered the fillers in certain areas. Apparently, polishing with PoGo did not generate sufficient abrasion for the HEMA matrix on the surface possibly due to the absence of water. Furthermore, polishing residues could be present in certain areas. This prevented the polishing material from being in direct contact with the nanoionomer surface. Visually, compared to the OptraPol group, the group polished with PoGo seemed to have a higher number of cracks. This may be caused by the absence of water, which causes the nanoionomer's dehydration. Overall, among all the polished groups, although PoGo exhibited the smoothest surface, the presence of HEMA matrix on certain areas of the surface, along with the presence of cracks, caused greater color changes in PoGo compared to OptraPol, but fewer ones compared to the control group and Sof-Lex XT Superfine.

Note that, the nanoionomer that was polished using OptraPol seemed to have fillers of regular sized shapes but with varying sizes. Furthermore, they were dispersed evenly on the surface while still being attached completely to the matrix. Among all the studied specimen groups, the exposed fillers on the surface seemed to be the largest. The nanoionomer surface polished using OptraPol seemed to be coarser than the surfaces of the samples that were polished using PoGo as well as the unpolished ones. However, the surface polished using OptraPol had the lowest number of cracks on the surface among all the polished groups. This may be because water's presence during the polishing process prevented the nanoionomer from dehydrating. Topographically, the nanoionomer that was polished with OptraPol seemed to have a coarser surface than that polished using PoGo or belonging to the control group. The exposed fillers on the surface resulted in lesser absorption of the coloring agent; however, HEMA matrix was still present between the fillers (it was required to keep them together). Thus, color changes were still present in this group due to the absorption of coloring agent by the HEMA matrix.

These results demonstrated that, compared to unpolished nanoionomers or nanoionomers polished using PoGo and Sof-Lex XT Superfine, polishing nanoionomers with OptraPol created surfaces that had a higher resistance to discoloration. Polishing with OptraPol could evenly abrade the HEMA matrix, exposing fillers to surface without causing it to detach. Prominences that were caused by exposed fillers on the nanoionomer surfaces created a coarse texture while preventing the absorption of coloring agents. However, hollow parts originating from the detached fillers, which resulted in a coarse surface, exhibit a higher coloring agent absorption tendency.

\section{CONCLUSION}

Based on this study, it can be concluded that polishing nanoionomers with Sof-Lex XT Superfine, PoGo, and OptraPol will lead to color changes. The smallest variations occurred in the material that was polished using OptraPol. Another important observation was that the immersion of unpolished and polished nanoionomers in a coffee-based solution will result into color changes, and the smallest variations, in this case, occurred for the nanoionomer that was polished using OptraPol.

\section{ACKNOWLEDGMENTS}

Publication of this manuscript is supported by Universitas Indonesia.

\section{CONFLICTS OF INTEREST}

All authors have none to declare.

\section{REFERENCES}

1. Uysal T, Yagci A, Uysal B, Akdogan G. Are nano-composites and nano-ionomers suitable for othodontic bracket bonding? Eur J Orthod 2010;32:78-82.

2. Sakaguchi RL, Power JM. Craig's Restotrative Dental Materials. $13^{\text {th }}$ ed. Philadelphia, PA: Elsevier; 2012. p. 186.

3. Philips RW. Skinner's Science of Dental Materials. $8^{\text {th }}$ ed. Philadelphia, PA: W.B. Saunders; 1982. p. 578-86.

4. Jefferies SR. Abrasive finishing and polishing in restorative dentistry: A state-of-the art review. Dent Clin North Am 2007:51:379-97.

5. Bouvier D, Duprez JP, Lissac M. Comparative evaluation of polishing systems on the surface of three aesthetic materials. J Oral Rehabil 1997;24:888-94.

6. Mallya PL, Acharya S, Ballal V, Ginjupalli K, Kundabala M, Thomas M. Profilometric study to compare the effectiveness of various finishing and polishing techniques in different restorative glass ionomer cement. J Interdiscip Dent 2013;3 Suppl 2:86-91.

7. Hartono. The World's Third Largest Coffee Production in the World. 2013. Available from: http://www.Kemenperin.go.id/artikel/6611/ Produksi-Kopi-Nusantara-Ketiga-Terbesar-Di-Dunia. [Last cited on 2014 Jun 07].

8. Ertaş E, Güler AU, Yücel AC, Köprülü H, Güler E. Color stability of resin composites after immersion in different drinks. Dent Mater J 2006;25:371-6.

9. Chan KC, Fuller JL, Hormati AA. The ability of foods to stain two composite resins. J Prosthet Dent 1980;43:542-5.

10. Gupta R, Parkash H, Shah N, Jain V. A spectrophotometric evaluation of color changes of various tooth colored veneering materials after exposure to commonly consumed beverages. J Indian Prosthodont Soc 2005;5 Suppl 2:72-8

11. 3M ESPE. Ketac TM. Technical Product Profile Ketac Cem Plus; 2005. Available from: http://www.multimedia.3m.com $/ \mathrm{mws} / \mathrm{media} / 367659 \mathrm{O} /$ ketac-cem-plus-technical-profile-in-english.pdf. [Last accessed on 2018 Aug 06].

12. Guler AU, Yilmaz F, Kulunk T, Guler E, Kurt S. Effect of different drinks om stainability of resin composite provesional restorative materials. J Prosthet Dent 2005;94 Suppl 2:118-24.

13. Schmitt VL, Puppin-Rontani RM, Naufel FS, Nahsan FP, Sinhoreti MA, Baseggio W. Effect of the polishing procedures on color stability and surface roughness of composite resins. ISRN Dent 2011;2011:617672.

14. Ghinea R, Ugarte-Alvan L, Yebra A, Pecho OE, Paravina RD, del Mar Perez M. Influrnce of surface roughness on the color of dental-resin 
composite. J Ahejiang Univ Sci B 2011;12 Suppl 7:552-62.

15. Bala O, Arisu HD, Yikilgan I, Arslan S, Gullu A. Evaluation of surface roughness and hardness of different glass ionomer cement. Eur J Dent 2012;6:79-86.

16. Sidhu SK, Pilecki P, SherriffM, Watson TF. Crack closure on rehydration of glass-ionomer materials. Eur J Oral Sci 2004;112 Suppl 5:465-9.

17. Naorungoj S. Effect of Surface Sealant on Staining Resistance of Novel Tooth-Colored Restoratove Materials. Chapel Hill: University of North Carolina; 2010. p. 39.
18. Padiyar N, Kaurani P. Color stabillity: An important physical property of esthetic restorative materials. Int J Clin Dent Sci 2010; Suppl 1:81-4.

19. Hattab F, Qudeimat M, Al-Rimawi H. Dental discoloration: An overview. J Esthet Dent 1999;11:291-310.

20. Preedy VR. Coffee in Health and Disease Prevention. $1^{\text {st }}$ ed. San Diego: Elsevier; 2014. p. 183.

21. De la Cruz AA, Hilbert G, Mengin V, Riviere C, Ollat N, Vitrac C, et al. Anthocyanin phytochemical profiles and anti-oxidant activities of Vitis candicans and Vitis doaniana. Phytochem Anal 2013;24:446-52. 